HUMANITASISayı - Number: 3

Bahar / Spring, Tekirdağ, 2014

\title{
HISTORIQUE DU ROMAN POLICIER TURC ${ }^{1}$
}

\begin{abstract}
Mustafa SOLMAZ ${ }^{2}$
Öz: Edgar Allan Poe'nun 1841 yılında yazdığ1 "Morg Sokağ Cinayetleri" adlı yapıtı dünya yazınında ilk polisiye roman olarak kabul edilir. Daha sonra, Arthur Conan Doyle'un Sherlock Holmes tiplemesi ilk özel dedektif olarak değerlendirilir. Polisiye roman, dünyada en çok okunan tür olmasına karşın, çoğu yazın adamı tarafindan yazınsal tür olarak kabul edilmez. Türk yazınında ilk çeviri polisiye roman, Ahmet Münif'in, 1881'de, Ponson du Terrail'in Les Tragédies (ou drames) de Paris adlı yapıtını Paris Faciaları adıyla yaptığı çeviridir. Üç yıl sonra Ahmet Mithat Efendi ilk yerli polisiye romanı Esrâr-ı Cinâyât'1 yazar. Tür, Osmanlı devletinin yıkılma aşamasında olmasından dolayı gelişim gösteremez, varlığını sürdürmekle yetinir. Batı yazınının etkisiyle ülkemize giren polisiye roman, Türk yazın geleneğinde yeni bir türdür. 130 yıllık kısa geçmişine karşın, son yıllarda, önemli başarılar göstererek Türk yazınında yeni, ancak çok önemli bir yer edinmiştir. 1980'li yıllara kadar konu sıkıntısı çeken Türk polisiye romanı, toplumun yaşam tarzının değişmesi, suç çeşitliliği ve oranının artmasıyla, derin devletten teröre, uyuşturucu kaçakçılığından karanlık cinayetlere kadar farklı konulara değinmeye başlamıştır. Özel televizyon kanallarının ortaya çıkışı ile miras, aile içi infaz, mafya, alacak-verecek sorunu gibi farklı konulara da değinilmeye başlamıştır. Son dönemde bazı polisiye roman yazarlarının yapıtları yabancı dillere çevrilmiştir. Yeni nesil polisiye roman yazarları sayesinde Türk polisiye romanı dünyaya açılma olanağı bulmaktadır.
\end{abstract}

Anahtar Sözcükler: Polisiye Roman, Batı Etkisi, Çeviri, Cinayet, Dedektif, Televizyon Dizileri, Toplum, Değişim.

\section{Introduction}

Le roman policier représente un genre relativement nouveau dans la tradition littéraire turque.

Le roman policier est un roman littéraire très pénible puisqu'il narre un événement principal où tous les personnages se trouvent et tournent autour de lui pour savoir celui qui l'a fait et les raisons qui l'ont poussés à le faire.

${ }^{1}$ Cet article s'est produit de la thèse de doctorat, s'intitulant L'Influence du Roman Policier Occidental Sur le Roman Policier Turc, soutenue en 2012 dans l'Université de la Manouba en Tunisie.

2 Yrd. Doç. Dr., Yüzüncü Y1l Üniversitesi, Yabanc1 Diller Yüksekokulu. mustafasolmaz@yyu.edu.tr 
Le roman policier est défini comme étant "le récit rationnel d'une enquête menée sur un problème dont le ressort principal est un crime" (Sadoul, 1988), c'est une définition qui correspond avant tout à ce que les théoriciens du genre désignent comme le récit d'énigme criminelle, représenté de façon symbolique dans l'œuvre d'Edgar Poe « Double assassinat dans la rue Morgue » en 1841.

C'est lui d'ailleurs qui a inventé le roman policier. Puis, Arthur Conan Doyle a créé le personnage de Sherlock Holmes considéré comme le prototype absolu du détective privé, Agatha Christie a gagné une grande popularité grâce aux personnages Hercule Poirot et Miss Marple.

L'école française du genre représentée par Maurice Leblanc et Gaston Leroux met en scène des héros plus marginaux comme Arsène Lupin, ou Joseph Rouletabille. Ces textes ont servi de modèle à des séries comme "Le club des cinq" ou "Alice".

On distingue trois catégories dans le genre de roman policier:

D'abord, le roman à énigme, qui est un roman ayant une structure narrative duelle où premièrement, deux histoires dans le roman existent, l'histoire du crime qui raconte ce qui s'est effectivement passé et l'histoire de l'enquête qui explique comment le lecteur ou le narrateur en a pris connaissance.

Deuxièmement, deux séries temporelles qui sont les jours de l'enquête qui commencent au moment du crime et les jours du drame qui mènent à lui. Les personnages principaux jouissent d'une immunité, rien ne peut leur arriver et le lecteur s'identifie au héros enquêteur. (Christie, 2003)

Ensuite, le roman noir, qui est différent dans sa structure et dans ses intentions, car il est inspiré du roman américain des années 30 (hard-boiled school - 1'école des durs à cuire), diffusé dans la série noire, c'est un roman de critique d'une société corrompue et désespérée. Il fusionne les deux histoires, il supprime la première et donne vie à la seconde, où le récit coïncide avec l'action.

Il se caractérise aussi par la différence dans ses thèmes où la violence (tabassage, massacre) est fort présente et l'amour est l'égal d'une passion désordonnée ou d'une grande haine.

On remarque aussi que le milieu représenté est différent avec le roman d'aventures qui contient aussi du danger, de la poursuite et du combat. (Lugarelli, 2002)

La seconde histoire qui se déroule au présent y tient une place centrale. Enfin, le roman à suspense ou thriller qui combine les deux premières catégories. En effet, il garde le mystère et les deux histoires du roman à énigmes, et donne la place centrale à la seconde histoire du roman noir.

Par conséquent, le lecteur est intéressé non seulement par ce qui est arrivé, mais aussi par ce qui va arriver plus tard; les deux types d'intérêt se trouvent réunis ici, car d'un côté, il y a la curiosité de savoir comment s'expliquent les événements passés, et d'un autre côté, il y a le suspense. C'est le cas des romans de Boileau-Narcejac, Mary Higgins Clark et de Patricia Highsmith. (Hoveyda) 
Le lecteur connaît mieux que le héros la source du mal, il est le témoin privilégié, mais impuissant, d'une histoire dont la fin paraît inévitable.

Le mystère a une fonction différente de celle du roman à énigmes, il est un point de départ, l'intérêt principal venant de la seconde histoire, celle qui se déroule au présent.

Devant tous ces genres du roman policier, on se demande: comment se positionne le roman policier turc?

Le roman policier turc est méconnu par le lecteur étranger jusqu'à douter de son existence.

Cependant, il marque sa présence par ses aspects particuliers qui lui procurent une place sur la scène de la littérature policière mondiale. D'ailleurs, c'est la problématique de notre présente recherche qui consiste à trouver l'existence et la persistance du roman policier turc.

Le roman policier apparaît dans les circonstances historiques et culturelles du $19^{\text {ème }}$ siècle. La ville industrielle constitue un des principaux facteurs de l'émergence du roman policier. Cette même ville synonyme de progrès et d'évolution, engendre aussi la misère, le danger et l'insécurité. Se confrontant à cette situation, la police réagit et rassure plus ou moins la population par sa présence, mais souvent elle compte plus sur les indicateurs pour arrêter le coupable que sur ses propres facultés intellectuelles. De l'autre côté, il y a le criminel qui échappe grâce au déguisement, à la loi et disparaît dans la foule une fois le crime accompli.

La presse et la science positive ont aussi été des facteurs d'émergence du genre. Aux journaux, il emprunte les récits de meurtres et il s'empare des méthodes scientifiques pour les besoins de l'intrigue. Dans toute affaire criminelle, il faut des preuves pour arrêter le coupable. L'interprétation des indices nécessite un discours cohérent, et logique argumenté.

De nos jours, un large public, surtout parmi les jeunes, lit et admire le roman policier qui suscite en même temps l'intérêt de plusieurs revues littéraires et fait l'objet de toutes sortes d'analyses critiques, de plus en plus fréquentes ces derniers temps. D'après Üyepazarc1, le roman policier occupe la troisième place parmi les livres en vente au monde après la Bible et "le livre rouge" de Mao Zedong. (Üyepazarc1, 1997)

Si le roman policier est né en Angleterre, puis s'est répandu en Amérique et en France. C'est après la deuxième guerre mondiale qu'il s'est étendu au reste du monde.

La parution du roman policier est très étroitement liée au développement de l'Etat de droit bourgeois.

La première forme du roman policier est apparue à travers le roman policier en feuilleton au $19^{\text {ème }}$ siècle. Il est publié sous la forme de feuilleton pour distraire les gens et leur faire oublier les problèmes et les difficultés de la vie quotidienne. 
Les premiers grands feuilletons sont publiés entre 1837 et 1850 par Ponson du Terrail auteur de "Rocambole". Toutes les composantes du roman policier se sont mises en place entre 1850 et la Première Guerre mondiale. (Reuter, 1997)

Comme genre, le roman policier a commencé, selon les historiens de la littérature, avec l'œuvre d'Edgar Allan Poe "Double Assassinat dans la rue Morgue", parue en avril 1841. Il a présenté la vraie histoire du crime avec son héros "le Chevalier Dupin" comme détective.

A la fin de l'année 1939, le style et les méthodes d'investigation du roman policier ont changé, car l'agent de police a pris la place du détective privé. On trouve de plus en plus de médecins légistes dans les laboratoires travaillant sur la cause de la mort et préoccupés par l'analyse des taches de sangs trouvées sur les habits des victimes, voire de particules de poussière recueillies sur les lieux du crime.

Après la deuxième guerre mondiale, le roman noir américain et le roman policier moderne ont gagné une grande popularité.

A cette époque, le roman policier français commence à se développer. Les thèmes s'élargissent et les romanciers utilisent de nouvelles techniques. Les écrivains du roman policier français commencent à créer un style original en liant la détente à l'analyse psychologique au sein de leurs romans.

Mais le roman policier a connu son âge d'or pendant les années vingt et les années trente, notamment en Amérique et en Angleterre.

Dès 1942, le roman noir français s'est reconstitué en trouvant un nouveau souffle avec Léo Malet. Il devient plus sensible aux idées politiques et plus précisément à l'idéologie socialiste. En effet, il est devenu un roman qui valorise le socialisme considéré comme libérateur de la société. En France, les événements de 1968 (maoïstes, trotskistes, gauchistes etc.), ont profondément influencé le développement du roman policier.

La première période du roman policier est marquée par l'importance de l'énigme, des indices, de l'enquête et du détective héros positif comme chez Conan Doyle et Agatha Christie.

La deuxième période est marquée plutôt par le roman psychosocial par Simenon.

Dans les troisième et quatrième périodes, l'angoisse, le suspense et la violence dominent le nouveau roman noir français surtout avec Boileau-Narcejac et Sébastien Japrisot.

En France, entre les années 1970 et 1980 une nouvelle tendance paraît avec des auteurs nés dans les années 1940. Ils veulent stabiliser le roman policier dans la réalité sociopolitique française, ce qu'ils considèrent être une littérature solidifiée. De toute façon la production s'est développée. Dans les années 1980, d'autres auteurs ont souvent partagé les mêmes objectifs que les écrivains de romans policiers tout en respectant les perspectives sociales et politiques. 
En effet, depuis son apparition et jusqu'à nos jours, le roman policier représente un outil de propagande pour les idées de ses auteurs. C'est ainsi que Dickens a défendu le policier, Poe a critiqué la capacité de la bourgeoisie à détruire le génie et l'originalité, Chesterton a fait l'apologie du catholicisme et Doyle l'apologie de la guerre, alors que Chester Himes s'est engagé dans la propagande politique dans la mesure où il était contre le racisme. (Roloff et Seesslen, 1997)

L'évolution du roman policier reflète l'histoire du délit dans un contexte intimement lié au cadre social, politique et idéologique de son époque. (Mandel -trad. Saraçoğlu-, 1996)

L'école française du genre représentée par Maurice Leblanc et Gaston Leroux met en scène des héros plus marginaux comme Arsène Lupin, ou Joseph Rouletabille. Ces textes ont servi de modèle à des séries comme "Le club des cinq" ou "Alice".

L'apparition d'une civilisation urbaine, et plus exactement d'une ville industrielle à la fin du $19^{\text {ème }}$ siècle, constitue l'une des circonstances de la naissance du roman policier.

La modernité du genre policier est constituée par deux facteurs interdépendants: la longévité du genre et son adaptabilité à l'évolution sociale.

Les premiers travaux sur l'histoire du roman policier commencent au début du $20^{\text {ème }}$ siècle. D'autre part, ils parlent aussi de l'histoire du genre, de la notion de policier et de la valeur de l'écriture du roman policier. Ces recherches, sont relatives à la sociologie littéraire et la plupart d'entre elles portent sur l'explication du genre. (Metzler et Nusser, 2003) La deuxième période de ce type de travaux a eu lieu durant les années 1930 et 1940 en Amérique et en Europe. La troisième période est entamée après les années 1960.

En Turquie, Üyepazarci Erol a écrit un essai, en 1997, sur le développement du roman policier et les informations générales sur les éditions traduites et produites du roman policier turc entre 1881 et 1928. En 2008, il a élargi le même travail de 1881 à 2006 dans deux volumes. Mais en Turquie, il n'y a aucun travail académique sur l'histoire du roman policier turc.

\section{L'influence de la modernité sur le genre}

Le genre policier se montre tout à fait ouvert aux nouvelles technologies. On trouve des criminels et des enquêteurs qui disposent de moyens de plus en plus sophistiqués ainsi que des lecteurs modernes qui peuvent même poursuivre leurs lectures sur Internet et participer à l'enquête interactive.

Le genre policier présente la particularité de distraire et de plaire en exploitant l'insécurité, en suscitant la peur, en se servant de cette forme d'excitation, de montée d'adrénaline, de stupéfaction qui s'empare de chacun des lecteurs face à un spectacle horrible. Les éditeurs l'ont compris, de même que les metteurs en scène et directeurs de programmes télévisés. 
Tout à fait en harmonie avec la société de consommation moderne, il prend la forme de produit jetable, à usage unique, vite oublié, vite remplacé.

Mais avant de décrire le roman policier turc, il faut parler de la proclamation de Tanzimat (les réformes administratives) en 1839, car après cet événement, la vie sociale de l'Empire Ottoman commençait à changer dans tous les domaines. La première organisation de police a été constituée en 1844. En 1846, l'expansion de cette institution étatique s'est élevée. L'organisation de la police secrète a aussi été constituée pendant ces années. Après 1856, les relations entre 1'Empire Ottoman et l'Occident s'intensifient. Certaines valeurs occidentales trouvent une bonne place parmi les Turcs et la situation sociopolitique change.

Les aspects culturels de l'occident, surtout ceux de la France, font leur entrée dans l'Empire Ottoman par l'élite turque. Le roman classique et le roman policier sont, par excellence, le reflet de ces aspects.

En Turquie, "Les Tragédies (ou drames) de Paris - Paris Faciaları" roman de Ponson du Terrail était le sujet de la première traduction du roman policier turc en 1881 faite par Ahmet Münif. (Üyepazarc1, 1997) Ce n'est qu'après trois ans de la traduction du premier roman d'enquête, qu'apparaît le premier roman policier turc en 1884 par Ahmet Mithat Efendi qui a écrit "Esrâr-1 Cinâyât - Les Secrets Des Crimes ".

Nous pouvons dire que le roman policier turc n'a pas pu se développer jusqu'à ces dernières années. Dès que le roman et le roman policier turc ont paru, ils ont été apréciés par le lecteur turc, mais, le roman policier turc n'a pas pu s'étendre à cause du déclin de l'Empire Ottoman. On remarque aussi que le motif du crime commis par le coupable turc ne constitue pas l'intrigue car le criminel n'utilise encore pas de gants et ne cherche pas à faire disparaître les traces du délit. En plus, le crime en général, se réalise devant des témoins et il y a donc beaucoup de preuves. C'est pour cela que le policier ne cherche pas le coupable par le biais des preuves; au contraire, il cherche les preuves par l'intermédiaire du coupable.

Entre 1881 et 1908 les traductions des romans policiers se faisaient en général du français vers le turc. A cette époque-là, le français était la langue de la culture et l'élite turque ne connaissait que le français comme langue étrangère.

Après 1908 on voit apparaître plusieurs traductions du roman policier. Presque tous les romans de grands écrivains occidentaux, fondateurs du genre du roman policier, ont été traduits.

La première traduction de Sherlock Holmes du célèbre écrivain Conan Doyle est "Dilenci - Mendiant" a été élaboré par Kirkor Faik en 1909. Puis, de nombreux traducteurs turcs ont traduit les aventures de Sherlock Holmes. Certains traducteurs ajoutent leurs opinions et leurs commentaires.

Tous ces éléments convergent pour créer une situation favorable aux changements au niveau des créations culturelles qui s'inspirent de l'Occident. 


\section{Les produits des romans policiers turcs}

Après avoir pendant des décennies traduit ou adapté des centaines d'aventures policières ou de séries d'enquête, les écrivains turcs se sont mis à écrire des fictions policières ancrées dans la réalité historique et sociale turque.

La première difficulté qu'ils vont rencontrer c'est bien celle de l'intrigue et du mystère qui entoure le crime pour que l'enquête ait de l'intérêt. Or, à cette époque les crimes n'étaient pas prémédités, donc l'intrigue manquait à l'histoire. Généralement, l'énervement, la jalousie ou bien le crime d'honneur sont le mobile des crimes commis. C'est pour cela que le coupable ne se cache pas et après avoir tué sans hésiter, il va directement au poste de police pour avouer son crime.

En Europe, sous l'effet de l'industrialisation, l'apparition de grandes villes et la métamorphose de la vie quotidienne sur le plan économique, politique, social et culturel au $18^{\text {ème }}$ siècle, le roman policier a vu le jour sous une autre forme plus vive et répondant aux besoins de l'époque et de la modernité naissante.

L'Occident a connu ce développement avant la Turquie. De nouveaux problèmes naissent dans les villes, l'organisation de la mafia fait son apparition, ainsi que les cambriolages, le banditisme, les pots-de-vin ont augmenté d'une manière inquiétante. Les conflits politiques et les problèmes sociaux n'en sont que le résultat. Aujourd'hui, la Turquie connaît ces mêmes problèmes, ce qui a contribué au développement du roman policier et explique son expansion.

Malgré la popularité des romans policiers en Turquie et leurs grands succès, on a rarement vu paraître des romans policiers turc durant de longues années. Ce n'est qu'en 1884 que Ahmet Mithat Efendi a écrit le premier roman policier turc "Esrâr-1 Cinâyât - Les Secrets Des Crimes". Dans ce roman, il a été influencé par Emile Gaboriau. Mais, il l'a écrit dans un style propre à lui et de temps en temps il a ajouté des explications et des informations pour les lecteurs. Par exemple dans un des romans il y a une scène de suicide, tout de suite il commence à parler des méfaits du suicide sur cinq pages. Il critique la corruption sociale, la justice, et l'administration, mais il donne raison à l'administration et la fait triompher en fin de compte pour ne pas s'attirer des ennuis.

Pendant des années, Ahmet Mithat Efendi était considéré comme un écrivain de référence pour les écrivains de roman policier turc. Ce système n'a pas changé et tous ceux qui ont écrit des romans policiers turcs ont suivi son modèle. Les écrivains glissent des informations encyclopédiques au cours du récit pour répandre davantage de culture générale auprès des lecteurs.

Durant les années 1914 à 1918, autrement dit pendant la Première Guerre Mondiale, il n'y avait aucune production de romans policiers turcs, parce que les auteurs, comme les autres citoyens turcs, étaient partis à la guerre.

Pour gagner leur vie, certains écrivains célèbres ont écrit des romans policiers. Peyami Safa qui est un romancier turc célèbre a aussi écrit des policiers en 
utilisant un pseudonyme "Server Bedi". Sa série noire "Cingöz Recai", a été un grand succès grâce à sa bonne adaptation des éléments psychologiques et sexuels aux normes du roman policier noir en 1924.

Le célèbre metteur en scène Metin Erksan a réalisé un film sur Cingöz Recai en 1954. Grâce à ce film, Cingöz Recai est devenu le premier héros du roman policier turc à évoluer dans un film.

Pendant les années 1930, certains écrivains turcs se sont consacrés à la littérature policière comme Cemil Cahit, Feridun Hikmet Es, Tahsin Abdi Gökşingöl, Süleyman Çapanoğlu.

Dans le monde entier, les années 40 ont été considérées comme étant l'âge d'or du roman policier.

La série de "Mike Hammer" occupe la première place parmi d'autres. Les maisons d'édition proposent au célèbre écrivain turc Kemal Tahir de traduire les romans de la série Mickey Spillane, il a en effet traduit six romans. $\mathrm{Vu}$ la demande du lectorat turc, les maisons d'éditions lui ont demandé d'écrire des romans sur "Mike Hammer". Personne n'a douté que ces romans ne sont pas l'œuvre de Mickey Spillane et la vente n'a pas diminué non plus. Kemal Tahir, Afif Yesari, Hayalet Oğuz ont aussi contribué à cette série qui a compté plus de 250 romans entre les années 1950-1960 en Turquie.

Néanmoins on remarque une diminution du nombre de romans policiers turcs dans les années 60 à cause de la haute tension politique.

Entre 1960-80, la tendance réaliste et socialiste a perdu son influence après le coup d'état militaire du 12 septembre 1980.

Le roman policier existe depuis 125 années en Turquie. Mais, ces dernières années, il a beaucoup gagné en popularité. En raison du développement économique et social de la Turquie et grâce à la croissance des richesses, la mentalité turque a automatiquement changé avec l'augmentation du revenu individuel.

De nos jours, à la différence de ce qui se passait avant, on peut voir des gens capables de tuer leurs parents pour l'héritage et de dissimuler leurs cadavres quelque part dans la maison familiale. Ce qui a été, d'ailleurs, une grande source d'inspiration pour les écrivains turcs. La vraie époque du roman policier turc commence dans les années 1980 suivant le rythme des évènements culturels.

Les années 1990 annoncent le commencement d'une époque glorieuse pour le roman policier turc. De nouveaux écrivains sont apparus et les maisons d'édition ont accordé beaucoup d'importance à la traduction des romans policiers étrangers. On trouve beaucoup d'articles sur le roman policier dans les revues et les livres littéraires tout en s'inspirant du trafic des œuvres antiques, de la sexualité, du chantage, des relations entre les politiciens, la mafia et la police, du trafic de la drogue, du satanisme et de la peur. 
Les romans policiers de certains écrivains turcs ont été traduits en langues étrangères comme les romans d'Orhan Pamuk, de Mine G. Kırıkkanat, d'Aslı Erdoğan et d'Ahmet Ümit. Nous pensons que le roman policier turc s'ouvrira au monde entier avec la nouvelle génération d'écrivains de romans policiers.

Orhan Pamuk, prix nobel de littérature, écrit "Benim Adım Kırmızı" "Mon nom est Rouge" en 1999 qui fait partie du roman policier postmoderne.

En Turquie, ces dernières années, le roman policier connaît un développement rapide et spectaculaire au niveau de l'élaboration technique. De nouveaux écrivains de romans policiers sont apparus comme Osman Aysu, Esmahan Aykol, İsmail Güzelsoy, Ferhat Ünlü, Emrah Serbes, Celil Oker, Mehmet Murat Somer, Birol Oğuz, Armağan Tunaboylu, Alper Canıgüz, Can Giray et Nihan Taştekin.

Le roman policier turc est un miroir de la société turque, de ses mutations, et de ses problèmes qui se reflètent dans les sujets des romans policiers.

Les romans policiers turcs étaient restés sous l'influence du roman policier et du cinéma occidental.

Pendant ces dernières années, le roman policier a connu un très grand succès chez les Turcs, car il a bien réussi à intégrer leur mode de vie et certaines de leurs coutumes.

C'est ainsi que les détectives turcs trouvent les assassins en respectant la mentalité et les habitudes turques.

En général, les écrivains turcs du roman policier relient la vie quotidienne aux événements de leurs romans et font progresser l'intrigue dans le respect des coutumes et des traditions turques, où les détectives turques arrêtent les criminels et reflètent leur culture qui est acceptée et adoptée par le lecteur turc.

Les romans thrillers sont populaires car ils parlent du développement de la politique internationale, des nouvelles technologies, des travaux scientifiques. Mais les écrivains turcs parlaient des cultures et du mode de vie turc. Cette popularité a incité les écrivains étrangers à les imiter ou à les adapter.

Après 1980, la nature des délits a changé et les crimes se sont diversifiés. En effet, la police est confrontée à beaucoup de crimes comme le blanchiment d'argent, l'exportation fictive, les réseaux d'intérêt, le terrorisme etc. Après 1990, les chaînes de télévision privées ont vu le jour et après un court laps de temps leur nombre a augmenté.

Les télévisions privées ont produit des séries qui traitent des sujets relatifs aux enquêtes policières comme les histoires d'espionnage, les secrets de famille, les tueurs en série, l'héritage, MIT (Milli Istihbarat Teşkilatı-Organisation nationale de renseignements), les exemples de la vie quotidienne, la police, la mafia, le satanisme etc.

Les motifs des crimes sont en général l'honneur, la vengeance, la dette et la créance, la fureur subite, l'effet de l'alcool, les problèmes familiaux, les relations amoureuses, l'hostilité entre les familles ou les personnes. 


\section{Conclusion}

Dans cette vague de traduction de la littérature turque de différents genres, le roman policier a su gagner sa place et se faire connaitre par le lecteur étranger à travers la traduction.

La réception de la littérature turque et du roman policier dans le monde devait surmonter plusieurs obstacles à sa traduction et à sa diffusion qui sont en relation directe avec la politique du pays et la culture turque.

Dans les romans policiers turcs, à part le détective privé, nous pouvons trouver l'agent de police, les curieux ainsi que les prototypes de certaines corporations professionnelles comme le professeur ou le journaliste. D'autres types peuvent encore s'y ajouter comme l'agent de police retraité ou n'importe quel autre individu à la place du détective.

Ces analyses nous permettent donc, de découvrir la forme du récit, l'identité et le statut du roman policier turc.

Il est toutefois difficile de prétendre avoir épuisé tout le problème. La recherche dans ce domaine est encore rudimentaire.

Pour ces raisons, le roman policier est finalement parvenu, après un siècle d'existence, à s'implanter au sein des espaces littéraires bien qu'il soit réputé comme genre mineur.

\section{BIBLIOGRAPHIE}

Auden, W. H. (1983) Autopsies du Roman Policier, «Le Presbytère coupable. Remarques sur le roman policier par un drogué », dans U. Eisenzweig. Paris: Union générale d'éditions.

Attila, İ. Erişim Tarihi: 9 Nisan 2008.

http://webarsiv.hurriyet.com.tr/2002/03/16/101363.asp.

Baudou, J. et Schléret, J-J. (2001). Le Polar. Paris: Larousse, Collection « Guide Totem ».

Baudou, J. (1986). Mystères 86. Paris: L.G. F. Coll. Le livre de Poche.

Bayraktar, E. (1998). 1884-1918 yılları arasında Türk edebiyatında polisiye roman. (Master tezi). Ankara: Gazi Üniversitesi Sosyal Bilimler Enstitüsü.

Bernhard, R. et Seesslen, G. (1997). Cinayet Sineması. İstanbul: Alan Yayınc1lik.

Benvenuti, S. et Rizzoni, G. (1982). Le roman criminel. Atalanta: Lebrun Michel.

Bezirci, A. et Taner, R. (1990). Seçme Hikâyeler (Nouvelles choisies). Istanbul: Kaya Yayınları.

Blanc, J-N. (1991). Polarville: images de la ville dans le roman policier. Lyon: Presses universitaires.

Boileau, P. et Narcejac, T. (1964). Le Roman policier. Paris: Payot. 
Bourdier, J. (1996). Histoire du roman policier. Paris: Editions de Fallois.

Boyer, A-M. (1988). Portrait de l'artiste en policier. Dans Modernités, (2). Presses Universitaires de Nantes.

Christie, Agatha (2003). Mort sur le Nil. (Ed.) Hachette. Paris: Collection Poche.

Çakır, Mikdat (2004). Savaş Sosyolojisi. Ankara: Mars Matbaası.

Çelenk S. (2005). Televizyon, Temsil, Kültür. 90’lı Yıllarda Sosyokültürel İklim ve Televizyon Içerikleri. Ankara: Ütopya Yayıncılık.

Ergil, D. (1984). Toplum ve Insan. "Toplumbilimin Temelleri”. Ankara: Turhan Kitabevi Yay.

Ferniot, C. (2001). La Folie du Polar. Dans Lire, (299), Octobre.

Fesch, P. (1971). Constantinople aux Derniers Jours d'Abdul-Hamid. Paris: M. Riviere.

Gezer, H. (2006). Türk Edebiyatında Polisiye Roman ve Ahmet Ümit'in Polisiye Roman Kurguları. Yüksek Lisans Tezi. Süleyman Demirel Üniversitesi.

Hoveyda, F. (1956). Petite histoire du roman policier. Paris: Ed. du Pavillon.

Iş1k, N. (Ağustos 1991). Şövalye romanlarından, pikareskten polis romanlarına, Varlik, (1007), İstanbul.

Küçükboyac1, M.-R. (1988). Ingiliz Edebiyatında Dedektif Hikâyeleri. İzmir: Ege Üniversitesi Edebiyat Fakültesi Yayınları.

Lugarelli, N. et C. (2002). Exemple: Jolies jambes. Collection Rat Noir (Ed.) Syros.

Mandel, E. (1996). Hoş Cinayet (Meurtres Exquis), Polisiye romanın toplumsal tarihi. (Çev. N.Saraçoğlu). İstanbul: Yazın Yayıncılık.

Metzler, J.B. et Nusser, P. (2003). Der Kriminalroman. Stuttgart.

Recatala, D. F.(1986). Le Polar. Paris: M. A. Editions.

Reuter, Y. (1997). Le roman policier. Paris: Edition Nathan.

Sadoul, J. (1988). Anthologie de la littérature policière. Paris: Ramsay.

Tilbe, A. (2008). Bir Polisiye Serüven Roman İncelemesi: Jean-Christophe Grangé'nin Kurtlar İmparatorluğu (Bildiri). A. Tilbe ve Ark. (Ed.). V. Ulusal Frankofoni Kongresi Kitabı, 25-26 Ekim 2007, 59-79. Erzurum: Bizim Büro Basımevi.

Üyepazarc1, E. (1997). Korkmayınız Mr Sherlock Holmes, Türkiye'de Polisiye Romanın 125 Yıllık Öyküsü (1881-1928). İstanbul: Göçebe Yayınları.

Vanoncini, A. (1993). Le roman policier. Paris: PUF. Que sais-je?

\section{THE HISTORY OF TURKISH DETECTIVE NOVEL}

Abstract: "The Murders in the Rue Morgue", written by Edgar Allan Poe in 1841, is considered to be the first detective novel. Afterwards, Arthur Conan Doyle's fictional character is accepted as the first detective. 
Although it is the most read kind of novel in the world, detective novel is not accounted as a literary genre by many critics. The first translated detective novel belongs to Ahmet Münif, who translated Ponson du Terrail's "Les Tragédies (ou drames) Paris" as "Paris Faciaları" in 1881. Three years later, Ahmet Mithat Efendi writes the first native detective novel "Esrâr-1 Cinâyât". The genre was not able to develop itself because of the decline of Ottoman Empire; it just continued its existence. Turkish detective novel, which is introduced to our country by the West, is a new kind in Turkish literature tradition. Despite its short past of 130 years, it displays a great success in Turkish literature recently. Having very restricted topics until 1980s, Turkish detective novel began to touch upon various topics including secret government issues, terrorism, drug smuggling, and dark murders. With the emerging of private television channels, various topics are added. In recent years some detective novel writers' works have been translated into foreign languages. Thanks to new detective novel writers, Turkish detective novels will be able to spread out its reputation to the world.

Keywords: Detective Novel, West Influence, Translation, Detective, Television Series, Society, Transformation. 